\title{
PRODUCCIÓN DE MARIGOLD (Tagetes patula cv. Durango orange) EN DIFERENTES MEDIOS DE CRECIMIENTO, BAJO CONDICIONES DE VIVERO DE LA UNIVERSIDAD NACIONAL AGRARIA LA MOLINA
}

\author{
PRODUCTION OF MARIGOLD (Tagetes patula cv. Durango orange) IN DIFFERENT \\ GROWING MEDIA AT NURSERY CONDITIONS IN THE NATIONAL AGRARIUM \\ UNIVERSITY, LA MOLINA.
}

\author{
${ }^{1}$ Juan Carlos Jaulis C. y ${ }^{1}$ Alejandro Pacheco
}

\begin{abstract}
Resumen
El objetivo de la presente investigación fue evaluar el crecimiento y desarrollo de Marigold (Tagetes patula cv. Durango orange) en diferentes medios de crecimiento en contenedores. Los tratamientos fueron: tierra de chacra-aserrín 1:1 (T1), tierra de chacra-compost 1:1 (T2), tierra de chacra-musgo 1:1 (T3), compost-aserrín 1:1(T4), compost-musgo 1:1 (T5), estiércol de caballo-aserrín 1:1 (T6) y estiércol de caballo-musgo 1:1 (T7). Se usó un diseño de bloques completos al azar (DBCA) con 3 repeticiones. Para la comparación de medias se utilizó la prueba estadística de Duncan a un nivel de significancia de 0.05 . Los resultados muestran que el mejor tratamiento fue compost-musgo (T5), que ofreció las mejores propiedades físicas y químicas para el buen crecimiento y desarrollo de Tagetes patula (Marigold) en las variables de crecimiento (altura de planta, número de hojas, diámetro de tallo y número de raíces) y rendimiento (número de flores) evaluadas. Los tratamientos T1, T4 y T6 presentaron los menores rendimientos, por efecto de la alta relación $\mathrm{C} / \mathrm{N}$ que le confirió el aserrín a los medios de crecimiento.
\end{abstract}

Palabras clave: Marigold, Tagetes patula, sustrato.

\begin{abstract}
Summary
The objective of this investigation was to evaluate the growth and development of Marigold (Tagetes patula cv. Durango Orange) in different growing media for containers. The treatments were: soil - sawdust 1:1 (T1), soil - compost 1:1 (T2), soil - moss 1:1 (T3), compost - sawdust 1:1 (T4), compost - moss 1:1 (T5), horse manure sawdust 1.1 (T6) and horse manure - moss 1:1 (T7). Plants were arranged in a randomized complete block design (RCBD) with three replications. Treatments means were compared with Duncan's test at significant level $(p=0.05)$. The best treatment was compost - moss (T5) which offered the best physical and chemical properties for the growth and development of Tagetes patula (Marigold) in growth variables (plant height, stem diameter and number of leafs and roots) and yield (number of flowers). The T1, T4 and T6 treatments had lower yields, for effect of the high relation $\mathrm{C} / \mathrm{N}$ of the sawdust in the growing media.
\end{abstract}

Key words: Marigold, Tagetes patula, substrate.

\section{Introducción}

El cultivo de plantas ornamentales en el Perú ha tomado mayor importancia en los últimos años, debido al interés por el cuidado y conservación del medio ambiente y su influencia en la mejora de la calidad de vida de la población. La Organización Mundial de la Salud recomienda $8 \mathrm{~m}^{2}$ por persona de áreas verdes, siendo Lima una de las ciudades que se encuentra por debajo de estos valores con $2.9 \mathrm{~m}^{2}$ de área verde per cápita(Instituto Metropolitano de Planificación, 2012), por ello las autoridades públicas han dispuesto políticas para la creación e implementación de espacios con vegetación donde las personas puedan ir a recrear de manera segura y saludable.
Los viveros públicos y privados han incrementado sus actividades de producción de plantas ornamentales en su afán de atender esta creciente demanda, esto ha llevado al mayor uso de insumos para la preparación de sustratos, siendo el musgo el mas requerido, sin embargo su alto precio se ha convertido en una desventaja para los viveristas, que buscan nuevas alternativas que puedan servir como medio de crecimiento para sus cultivos y al mismo tiempo les permitan reducir y minimizar sus costos de producción. Ante esta situación se opta por otras alternativas como: estiércol de caballo, cascarilla de arroz, compost, cascarilla de café, aserrín, entre otros, insumos que al mezclarlos en proporciones adecuadas pueden servir como sustrato. 
Las plantas ornamentales pueden ser apreciadas por su follaje o sus flores, pudiendo ser de estación o perennes, la mayoría de estas provienen de zonas tropicales mostrando sensibilidad a sales y exigencias en agua. Entre las plantas de estación más utilizadas en el diseño de áreas verdes urbanas en la ciudad de Lima se tiene al Marigold (Tagetes patula cv. Durango orange). Según su clasificación taxonómica pertenece al reino; Plantae; División: Magnoliophyta; Clase: Magnoliopsida; Orden: Asterales; Familia: Asteraceae; Tribu: Tageteae; Género: Tagetes; Especie: patula (Gilman y Howe, 1999). El marigold es una planta herbácea anual, de porte erecto, consistente, textura media, cuya altura de la planta va a depender del cultivar $(0.15 \mathrm{~m}$ hasta los $0.90 \mathrm{~m}$.), y se consideran plantas de excelente cobertura y crecimiento (Sagastegui, 2004). Para su crecimiento requiere de suelos franco-arenosos con abundante materia orgánica, pH 6.5 a 7.0 (Tutan y Crouse, 1997), Conductividad eléctrica de 4 a $5 \mathrm{dS} . \mathrm{m}^{-1}$ siendo moderadamente tolerante a la salinidad (Tanji et al. 2007; Valdez et al. 2009). Así mismo, Jaurón (1996) recomienda suelos con buen drenaje.

\section{Sustratos}

Un sustrato es todo material natural o artificial, que permite el anclaje del sistema radicular de una planta y que proporciona nutrientes (Guerrero, 1993; Crozon y Neyroud, 1990; Lemaire et al. 2005) y puede presentarse en forma pura o en mezcla con un volumen constante (Abad, 1991).

Un buen sustrato debe mantener un equilibrio entre la fase solida, que constituirá el soporte físico del vegetal, la fase liquida que proporcionará agua y nutrientes, y la fase gaseosa que aportará una buena oxigenación a las raíces (Foucard, 1997). Las propiedades físicas de un sustrato son las más importantes, pues son muy difíciles de modificar, mientras que las propiedades químicas si pueden ser alteradas, inclusive posteriormente al establecimiento del cultivo (Abad, 1991). Es necesario conocer el $\mathrm{pH}$ ideal y como cambiara la cantidad de sales solubles presentes en el sustrato, así como la disponibilidad de los nutrientes para el desarrollo de plántulas (Fonteno 1998, Lemaire et al. 2005).

Abad (1993), menciona que las propiedades físicas deseables en un sustrato ideal son: una elevada capacidad de retención de agua, suficiente suministro de aire, buena distribución de tamaño de partículas, baja densidad aparente, elevada porosidad total, estructura estable y contracción reducida. Dentro de las propiedades químicas estas deben de caracterizarse por: presentar moderada a elevada capacidad de intercambio catiónico superior a 20 meq. $100 \mathrm{~g}^{-1}$, balance de nutrientes asimilables, baja salinidad, pH moderadamente ácido, elevada capacidad tampón, mínima velocidad de descomposición; y otras propiedades como: bajo costo, resistencia a cambios físicos, químicos y ambientales extremos, fácil de mezclar, humectar y desinfectar, reproductibilidad y disponibilidad, libre de semillas de malezas,nematodos y otros patógenos.

La relación $\mathrm{C} / \mathrm{N}$ es otro factor importante a tener en cuenta. Las materias orgánicas pasan por un proceso de descomposición antes de su empleo como sustrato, sin embargo si este no es adecuado se producirán fenómenos fitotóxicos y la inmovilización de nitrógeno (Ansorena, 1994). La cantidad de carbono mineralizado depende sobre todo de la naturaleza de los compuestos carbonados del material, más que la relación $\mathrm{C} / \mathrm{N}$; los materiales ricos en lignina se descomponen muy lentamente, al contrario que los materiales ricos en celulosa y hemicelulosa (Bunt, 1976).

\section{Insumos para sustratos}

El aserrín, es un material usado ampliamente en la preparación de sustratos, pero hay que agregarle nutrientes complementarios. Frente a problemas fitopatológicos, el aserrín es un sustrato supresivo para el desarrollo de Phytophthora, debido a que mejora el drenaje eliminando condiciones de anaerobiosis necesarias para el desarrollo de este hongo (Owney et al. 1990). El pH del aserrín fresco puede oscilar entre 4.5 -5.5 y aumenta hasta 6.5 7.0 cuando se composta.

El compost es de alto valor para los cultivos en viveros e invernaderos, sobre todo para los sustratos de enraizamiento, sus características dependen de la cantidad y tipo de insumos utilizados en su preparación, así como las condiciones ambientales que dominaron el proceso de descomposición (Guerrero, 1993).

La turba, resulta de la descomposición incompleta de la materia orgánica en condiciones de excesiva humedad y deficiencia de aire. Toda turba se descompone de restos vegetales no humificados, mantillo y minerales (Yagodín, 1986). Su principal uso es por su alta capacidad de retención de humedad en suelos ligeros o muy permeables, por lo que se le considera como un mejorador de las propiedades físicas del suelo. Su aporte de nutrientes es pobre (Guerrero, 1993).

El estiércol aporta a los suelos nutrientes, incrementa la retención de humedad y mejora la actividad biológica, todo esto conlleva a incrementar la productividad del suelo (Yagodín, 1986). El estiércol seco contiene en promedio: nitrógeno $1.57 \%$, fósforo $0.40 \%$, potasio $2.43 \%$ y conductividad eléctrica de 20.70 dS.m ${ }^{-1}$. Muchos de estos nutrientes están en forma orgánica y son lentamente disponibles para las plantas. También contiene calcio, magnesio, azufre y alguna cantidad de elementos menores (Palacios, 2007).

La tierra de chacra está compuesta por arena, limo y arcilla que es la parte mineral y un porcentaje mínimo de materia orgánica, no es uniforme, pues esto varía de acuerdo al material parental del cual se originó. Su clasificación va de acuerdo a la clase textural. 


\section{Sustratos para contenedores}

En zonas productoras de plantas ornamentales de mediante lavado y venteado respectivamente. Las Lima, como Lurín, los insumos más utilizados en medios de crecimiento son: tierra de chacra, arena, estiércol, musgo, aserrín y viruta. La proporción de mezcla es determinada por cada vivero. (Anca, 2003).

Para tomar la decisión de utilizar un material como sustrato, se debe considerar el costo, disponibilidad, cuidado del medio ambiente y que los resultados de la caracterización física, química y biológica se ajusten en lo posible a las características ideales para el crecimiento y desarrollo del cultivo a producir (Cruz et al. 2012).

Medina et al. (2011) mencionan que el desafío de la horticultura moderna es la obtención de una planta con una buena relación entre el sistema radicular y la parte aérea para lograr una buena adaptabilidad en el campo. El desarrollo y formación de nuevos tejidos es promovida por moléculas de ATP producidas durante la respiración aeróbica que rinde 38 ATP a partir de una molécula de glucosa, si en el medio existe poca disponibilidad de oxigeno (encharcamiento) se producirá la fermentación produciéndose solo 2 ATP, por lo tanto el rendimiento energético será menor y perjudicara el desarrollo de las raíces.

El presente trabajo, tiene como objetivo evaluar el crecimiento y desarrollo de Marigold (Tagetes patula $\mathrm{cv}$. Durango orange) en diferentes medios de crecimiento en contenedores, bajo condiciones de vivero.

\section{Materiales y métodos}

El desarrollo de la investigación se llevó a cabo en el tinglado del Vivero de Plantas Ornamentales de la Universidad Nacional Agraria La Molina.El distrito de La Molina geográficamente se encuentra a una latitud de $12^{\circ} 06^{\prime} \mathrm{S}$, longitude $76^{\circ} 57^{\prime} \mathrm{O}$ y altitud 243.7 m.s.n.m. y está ubicado dentro de un desierto subtropical árido aluroso según el sistema modificado de Koppen, con temperatura media anual de $18.5^{\circ} \mathrm{C}$, radiación anual de $186.5 \mathrm{cal}-\mathrm{g} / \mathrm{cm} /$ día, humedad relativa anual de $85 \%$ en peso y precipitación anual acumulada de $6 \mathrm{~mm}$.

Se realizó un análisis de caracterización a los insumos utilizados en la investigación en el Laboratorio de Análisis de Suelos, Plantas, Aguas y Fertilizantes (LASPAF) de la Facultad de Agronomía de la UNALM. Para la preparación de los sustratos se utilizaron los siguientes insumos: compost, musgo, tierra de chacra, aserrín y estiércol de caballo que son comúnmente utilizados en la producción de plantas ornamentales en el Perú. Los insumos se mezclaron en proporciones de 1:1 para la preparación de los sustratos. Los insumos aserrín $\mathrm{y}$ estiércol de vacuno fueron previamente tratados características de los insumos se muestran en el Tabla 1. 
Tabla 2. Resumen de las características de los sustratos evaluados.

\begin{tabular}{|c|c|c|c|c|c|c|c|c|c|}
\hline Tratamientos & Proporción & $\mathrm{pH}$ & $\begin{array}{c}\mathrm{CE} \\
\mathrm{dS} \cdot \mathrm{m}^{-1}\end{array}$ & $\begin{array}{l}\text { Meq. } \\
100 g^{-1}\end{array}$ & $\underset{\%}{\mathrm{MO}}$ & $\begin{array}{c}\text { DA } \\
\text { g.cm }{ }^{-3}\end{array}$ & $\begin{array}{c}\text { DR } \\
\text { g.cm }{ }^{-3}\end{array}$ & $\begin{array}{c}\text { D } \\
\text { g.cm } \\
-3\end{array}$ & $\underset{\%}{\mathrm{MRH}}$ \\
\hline Tierra de chacra-aserrín (T1) & $1: 1$ & 7.33 & 3.04 & 11.20 & 17.21 & 0.65 & 1.88 & & 64.43 \\
\hline Tierra de chacra-compost (T2) & $1: 1$ & 7.28 & 5.17 & 18.08 & 8.19 & 0.84 & 2.18 & & 90.65 \\
\hline Tierra de chacra- musgo (T3) & $1: 1$ & 7.36 & 3.65 & 11.52 & 1.84 & 1.00 & 2.18 & & 87.58 \\
\hline Compost-aserrín (T4) & $1: 1$ & 7.41 & 3.09 & 32.48 & 45.16 & & & 0.36 & 174.56 \\
\hline Compost-musgo (T5) & $1: 1$ & 7.11 & 3.52 & 40.00 & 37.85 & & & 0.21 & 202.45 \\
\hline Est. de caballo-aserrín (T6) & $1: 1$ & 8.13 & 11.1 & 30.80 & 65.25 & & & 0.20 & 217.67 \\
\hline Est. de caballo-musgo (T7) & $1: 1$ & 8.00 & 15.7 & 45.60 & 58.79 & & & 0.10 & 301.81 \\
\hline
\end{tabular}

Fuente: Laboratorio de Análisis de Suelos, Plantas, Aguas y Fertilizantes del departamento de Agronomía de la UNALM (2009).

$\mathrm{CE}=$ conductividad eléctrica, $\mathrm{CIC}=$ capacidad de intercambio catiónico, $\mathrm{MO}=$ materia orgánica, $\mathrm{DA}=$ densidad aparente, $\mathrm{DR}=$ densidad real, $\mathrm{D}=$ densidad, $\mathrm{MRH}=$ máxima retención de humedad.

\section{Resultados y discusión}

\section{Variables de crecimiento.}

De acuerdo a los análisis de varianza realizado se han encontrado diferencias estadísticas altamente significativas en todas las variables analizadas. Los resultados del efecto de los diferentes sustratos evaluados en Tagetes patula cv. Durango orange, se muestran en el Tabla 3, donde se observa de acuerdo a la prueba de comparación de medias Duncan, que el tratamiento compost-musgo (T5) fue superior en cada una de las variables evaluadas; sin embargo en altura de planta y diámetro de tallo no tuvo diferencias con los tratamientos T2, T3 y T7, en el número de hojas no las tuvo con el T2 y en el número de raíces con el T3.

Los resultados del tratamiento (T5) se explican en el hecho que las plantas encontraron las condiciones adecuadas para expresar su potencial genético (AzcónBieto y Talón, 2000). Respecto al sustrato, el pH resultó ligeramente alcalino (7.11), siendo el que más se acercó al pH ideal recomendado por Abad (1993) facilitando la disponibilidad de nutrientes presentes en el sustrato. La presencia de sales fue de $3.52 \mathrm{dS} .^{\mathrm{m}-1}$, lo que no afectó el desarrollo de las raíces. La capaçidad de intercambio catiónico fue de 40 meq. $100 \mathrm{~g}$, siendo superior a lo recomendado por Abad (1993) para el cultivo en sustratos. Su máxima retención de humedad fue de $202.45 \%$, lo que facilitó el drenaje del exceso de agua del espacio poroso, permitiendo el intercambio gaseoso para una buena respiración del sistema radicular. Según Lemaire et al. (2005) la mezcla compost-musgo tiene una relación $\mathrm{C} / \mathrm{N}$ de 14 y 20, siendo este sustrato más inestable y de pronta mineralización, contribuyendo con la liberación de nutrientes en la solución. El tratamiento (T5) estuvo conformado por compost que es una materia orgánica próxima a la humificación y mineralización, y musgo que se obtuvo de zonas alto andinas frías (10 ${ }^{\circ} \mathrm{C}$ ) y húmedas, incluyendo sus raíces con partículas de suelo del lugar en el que se desarrollaron. Al mezclar estos dos insumos, para ser utilizados como sustrato bajo condiciones del ensayo (temperaturas de 25.7 ${ }^{\circ} \mathrm{C}$, buena aireación y aporte de carga microbiana por parte del compost) contribuyeron a mejorar el proceso de descomposición, humificación y mineralización de la mezcla, facilitando la disponibilidad de nutrientes necesarios para la planta en la solución acuosa. Oberpaur et al. (2010), obtuvo resultados similares en el cultivo de lechuga con sustratos que estuvieron compuestos por mezclas de musgo-humus (60-40\%) y musgo-compost $(60-40 \%)$, siendo estas proporciones parecidas a la utilizadas en el cultivo de Marigold.

Sin embargo Valenzuela et al., (2003) encontró que cuando el sustrato estuvo compuesto solo por compost, las características en tamaño de Tagetes patula no fueron buenas; esto se debió a que el compost presento un $\mathrm{pH}$ alcalino, perjudicando la disponibilidad de nutrientes.

Los tratamientos tierra de chacra-musgo (T3) y tierra de chacra-compost (T2) fueron los que ocuparon el segundo $\mathrm{y}$ tercer lugar en general en las variables de crecimiento evaluadas, estos resultados se dieron porque dentro de su formulación tuvieron como uno de sus ingredientes al musgo y compost respectivamente, siendo estos insumos los que ayudaron a mejorar las características físicas y químicas del medio de crecimiento.

Los tratamientos tierra de chacra-aserrín (T1), compostaserrín (T4) y estiércol de caballo- aserrín (T6) presentaron un $\mathrm{pH}$ de $7.33,7.41$ y 8.13 respectivamente, provocando la restricción de nutrientes en el medio acuoso. No se realizó análisis de relación $\mathrm{C} / \mathrm{N}$ de los sustratos utilizados, sin embargo de acuerdo a los resultados obtenidos se asume, que la presencia de aserrín en estos tratamientos caracterizó a los medios de crecimiento con una alta relación $\mathrm{C} / \mathrm{N}$ de 80-150 (Stoffella \& Kahn, 2005), originando la competencia de los microorganismos descomponedores de la materia orgánica con la raíz por tomar el nitrógeno, teniéndose en estos tratamientos los valores más bajos en todas las variables de crecimiento evaluadas. Así mismo en el tratamiento estiércol de caballo-aserrín (T6) influyo también la presencia de sales que provocó desórdenes fisiológicos, desequilibrios iónicos, disminución en el potencial hídrico del medio nutritivo y restricción de la absorción de agua por las raíces. 
Tabla 3. Variables de Crecimiento de Tagetes patula cv. Durango orange evaluadas en siete diferentes sustratos: Altura de planta (Ap), $\mathrm{N}^{\circ}$ de hojas por planta $\left(\mathrm{N}^{\circ} \mathrm{h}\right)$, Diámetro de tallo de planta (Dt) y Número de raíces por planta $\left(\mathrm{N}^{\circ} \mathrm{r}\right)$.

\begin{tabular}{lccccc}
\hline \multicolumn{1}{c}{ Tratamientos } & $\mathrm{T}$ & $\begin{array}{c}\mathrm{Ap} \\
(\mathrm{cm})\end{array}$ & $\begin{array}{c}\mathrm{N}^{\circ} \mathrm{h} \\
(\mathrm{und})\end{array}$ & $\begin{array}{c}\mathrm{Dt} \\
(\mathrm{cm})\end{array}$ & $\begin{array}{c}\mathrm{N}^{\circ} \mathrm{r} \\
(\mathrm{und})\end{array}$ \\
\hline Tierra de chacra- aserrín & $\mathrm{T} 1$ & $14.78 \mathrm{c}$ & $37 \mathrm{~d}$ & $0.61 \mathrm{c}$ & $2.33 \mathrm{e}$ \\
Tierra de chacra-compost & $\mathrm{T} 2$ & $16.26 \mathrm{bc}$ & $189 \mathrm{ab}$ & $0.93 \mathrm{a}$ & $6.55 \mathrm{bc}$ \\
Tierra de chacra-musgo & $\mathrm{T} 3$ & $16.78 \mathrm{abc}$ & $151 \mathrm{c}$ & $0.97 \mathrm{a}$ & $7.89 \mathrm{ab}$ \\
Compost-aserrín & $\mathrm{T} 4$ & $16.36 \mathrm{bc}$ & $60 \mathrm{~d}$ & $0.77 \mathrm{~b}$ & $3.55 \mathrm{de}$ \\
Compost-musgo & $\mathrm{T} 5$ & $18.52 \mathrm{ab}$ & $213 \mathrm{a}$ & $1.05 \mathrm{a}$ & $8.22 \mathrm{a}$ \\
Estiércol de caballo-aserrín & $\mathrm{T} 6$ & $14.84 \mathrm{c}$ & $60 \mathrm{~d}$ & $0.76 \mathrm{~b}$ & $3.88 \mathrm{~d}$ \\
Estiércol de caballo-musgo & $\mathrm{T} 7$ & $18.02 \mathrm{a}$ & $170 \mathrm{bc}$ & $1.03 \mathrm{a}$ & $5.78 \mathrm{c}$ \\
\hline Cada par de tratamientos con la misma letra no son signiticativos al nivel de $0.05 \%$ (Prueba de Rango Multiple Duncan). Ap= altura de planta, $\mathrm{N}=$
\end{tabular}
número de hojas por planta, $\mathrm{Dt}=$ diámetro de tallo por planta, $\mathrm{N}^{\circ} \mathrm{r}=$ número de raíces por planta.

\section{Variable de rendimiento}

Realizado el análisis de varianza de la variable de rendimiento, de manera similar a las variables de crecimiento, también se encontró diferencias altamente significativas entre los tratamientos evaluados (Tabla 4). Siendo el tratamiento compost-musgo (T5) el que obtuvo el mayor número de flores con 5.25 pero sin diferencias significativas con los tratamientos tierra de chacracompost (T2), tierra de chacra-musgo (T3) y estiércol de caballo-musgo (T7), teniendo una relación directa con los resultados de las variables de crecimiento evaluadas. No se consideró la evaluación de la variable calidad de la flor, sin embargo se observó que el T5 fue el que presentó las mejores características.

Los tratamientos tierra de chacra-compost (T2), tierra de chacra-musgo (T3) y estiércol de caballo-musgo (T7) ocuparon el segundo, tercer y cuarto lugar respectivamente después del T5. Esto se explica en el hecho de que los sustratos dentro de sus mezclas tenían como insumo común al compost y/o musgo que ayudó a mejorar las propiedades físicas, químicas y biológicas del medio.

Tabla 4. Variable de Rendimiento de Tagetes patula cv. Durango orange evaluadas en siete diferentes sustratos: Número de flores por planta $\left(\mathrm{N}^{\circ} \mathrm{f}\right)$.

\begin{tabular}{|c|c|c|}
\hline Tratamientos & $\mathrm{T}$ & $\begin{array}{l}\mathrm{N}^{\circ} \mathrm{f} \\
\text { (und) }\end{array}$ \\
\hline Tierra de chacra- aserrín & $\mathrm{T} 1$ & $1.25 \mathrm{c}$ \\
\hline Tierra de chacra-compost & $\mathrm{T} 2$ & $4.91 \mathrm{a}$ \\
\hline Tierra de chacra-musgo & $\mathrm{T} 3$ & $4.75 \mathrm{a}$ \\
\hline Compost-aserrín & $\mathrm{T} 4$ & $2.75 \mathrm{~b}$ \\
\hline Compost-musgo & T5 & $5.25 \mathrm{a}$ \\
\hline Estiércol de caballo-aserrín & T6 & $1.83 \mathrm{bc}$ \\
\hline Estiércol de caballo-musgo & $\mathrm{T} 7$ & $4.75 \mathrm{a}$ \\
\hline
\end{tabular}

Los tratamientos T1 (tierra de chacra-aserrín), T4 (compost-aserrín) y T6 (estiércol de caballo-aserrín) que tienen como insumo en común al aserrín son los que menor rendimiento presentaron. Según Stoffella \& Kahn
(2005) el ingrediente aserrín tiene una alta relación C/N 80-150, por lo que para descomponer esta materia orgánica empezarán a proliferar los microorganismos, cuyos tejidos tienen una relación $\mathrm{C} / \mathrm{N}$ del orden de 30 , y si el material orgánico a descomponer tiene una mayor relación $\mathrm{C} / \mathrm{N}$, estos necesitarán un aporte extra de nitrógeno, por lo tanto tomarán el nitrógeno soluble del medio de crecimiento compitiendo con las plantas por este nutriente esencial (Ansorena, 1994). Dándose como resultado un menor crecimiento y por ende menor número de flores en estos tratamientos. Sin embargo Ortega et al., 2010 encontró que el sustrato aserrín presentó efectos similares a la turba en cuanto a altura, diámetro de tallo y mayor peso seco; esto puede deberse a que el aserrín que se utilizó en ese ensayo fue compostado previamente, teniendo en el medio de crecimiento un baja relación $\mathrm{C} / \mathrm{N}$.

\section{Conclusiones}

El tratamiento compost-musgo (T5) en general es el que brindó las mejores propiedades físicas y químicas para el crecimiento y productividad de la planta ornamental Tagetes patula cv. Durango orange, con una altura de planta de $18.02 \mathrm{~cm}, 213$ hojas, $1.05 \mathrm{~cm}$ de diámetro de tallo, 7.89 raíces y 5.25 flores por planta.

Los tratamientos tierra de chacra-aserrín (T1), compostaserrín (T4) y estiércol de caballo- aserrín (T6), son los que presentaron los menores valores en las variables de crecimiento y rendimiento evaluadas, esto se debió a la alta relación $\mathrm{C} / \mathrm{N}$ que aportó en todos ellos el insumo aserrín, produciéndose en el medio de crecimiento competencia por el nitrógeno entre los microorganismos y la planta.

\section{Literatura citada}

Abad, M.; Noguera, P.; Puchades, R.; Maquieira, A. and Noguera, V. 2004. Physico- chemical and chemical properties of some coconut coir dusts for use as a peat substitute for containerised ornamental plants. Biores. Technol. 82:241-245.

Abad, M. 1991. Los Sustratos Hortícolas. In: II Congreso Nacional de Fertirrigación. Almería, 18 - 20 septiembre. Fundación para la Investigación Agraria en la provincia de Almería. ESP. 
Abad, M. 1993. Sustratos. Características y propiedades. En: Cultivos Sin Suelo. F. Canóvas y J.R. Díaz. (eds.). Instituto de Estudios Almerienses. FIAPA. Pag. 47-62.

Anca, S. 2003. Producción y comercialización de plantas ornamentales en la zona sur de Lima. Tesis. Universidad Nacional Agraria la Molina. Lima. PE. 63 pp.

Ansorena, M. 1994. Sustratos: Propiedades y caracterización. Ediciones Mundi Prensa. ESP. 172 pp.

Azcón-Bieto, J. y Talón, M. 2000. Fundamentos de fisiología vegetal. Ediciones Universidad de Barcelona. McGraw-Hill Interamericana. ESP.

Bunt, A. C. 1976. Modern potting compost. A manual on the praparation and use of growing media for pot plants. Ed. George Allen and Unwin Ltd. Londres. 277 pp.

Crozon, J. and Neyroud, J. 1990. Etude des caracterítiques physiques de quelques substrats en horticulture. Review Suisse. Viticulture, Arboriculture, Horticultura.

Cruz, C.; Can C.; Sandoval, V.; Bugarín, M.; Robles, B. y Juárez, L. 2012. Sustratos en la horticultura. Revista Biociencias. MX. Pag. 2 (2) 17-26.

Fonteno, W. 1998. Know your media: the air, water and container connection. Grower Talks. V. Ball publishing. Pag. 110-111.

Foucard, J. 1997. "Viveros. De la producción a la plantación". Ediciones Mundi Prensa. Barcelona. ESP. 439 pp.

Guerrero, J. 1993. Abonos orgánicos. Tecnología para el manejo ecológico de suelos. Lima. PE. 189 pp.

Gilman, E. and Howe, T. 1999. Tagetes erecta L. Fact. Sheet FPS -569. Enviromental Horticulture Departament, Florida Cooperativa Extensión Service. Instituto of Food and agricultural sciences. Universidad of Florida.

Instituto Metropolitano de Planificación. 2012. Plan regional de desarrollo concertado de Lima (2012-2025). 441 pp.

Jaurón, R. 1996. Marigold Departament of Horticulture. Iowa State University. 22 pp.

Laboratorio de Análisis de Suelos, Plantas, Aguas y Fertilizantes del departamento de Agronomía de la Universidad Nacional Agraria La Molina. 2009.

Lemaire, F.; Dartigues, A.; Riviére, L.; Charpentier, S. y Morel, P. 2005. Cultivos en macetas y contenedores, Principios agronómicos y aplicaciones. Ediciones Mundi Prensa. MX. Pag. 109-112.

Medina, C.; Furlani, P. y Bataglia, O. 2011. Nutrição mineral e arquitectura do sistema radicular de mudas. Revista Plasticultura. BR. Ano V. N 19.

Mengel, K.y Kirkby, A. 2000. Principios de Nutrición Vegetal. Editor International Potash Institute. Suiza. 692 pp.
Oberpaur, C.; Puebla, V.; Vaccarezza F. and Arévalo, M. 2010. Preliminary substrate mixtures including peat moss (Sphagnum magellanicum) for vegetable crop nurseries. Cien. Inv. Agr. 37(1):123-132.

Ortega, M.; Sánchez, J.; Díaz, R. y Mendoza, J. 2010. Efecto de diferentes sustratos en el crecimiento de plántulas de tomate (Lycopersicum esculentum M.). Ra Ximbai, vol. 6 núm. 3. Set-dic. MX. Pag. 365-372.

Owney, B.; Benson, D. and Bilderback, T. 1990. Physical properties of container media and relation to severity of phytophthora root rot of Rhododendron. J. Amer. Soc. Hort. Sci. N 115 (4): 564-570.

Palacios, J. A. 2007. Manual de manejo de viveros. Universidad Nacional Agraria La Molina. 175 pp.

Sagastegui A. A. 2004. Descripción botánica de marigold (Tagetes erecta L.). Trujillo. PE.

Stoffell, P. y Kahn, B. 2005. Utilización del compost en los sistemas de cultivo hortícola. Ediciones MundiPrensa. Madrid, Barcelona. 26 pp.

Tanji, K.; Grattan, S.; Grieve, C.; Harivandi, A.; Rollins, L.; Shaw, D.; Sheikh, B. and Wu, L. 2007. Salt management guide for landscape irrigation with recycled water in coastal southern California. Editor Davis. University of California. 320 pp.

Tutan, D y Crouse, K. 1997. pH Soil and fertilizers. Mississippi State University. 117-119 pp.

Valdez, L.; Grieve, C. and Poss, J. 2009. Salinity and alkaline in irrigation water affect

Marigold plants: I. Growth and shoot dry weight partitioning. HortScience 44(6):1719-1725.

Valenzuela, O; Nicolau, F.; Rode, M.; Schlund, H y Gallardo C. 2003. Respuesta de Tagetes patula a sustratos formulados con compost y perlita. En: Revista científica agropecuaria 7(1):57-61. Facultad de ciencias agropecuarias UNER.

Yagodín, B. 1986. “Agroquímica”. Editorial Mir Moscú. URSS. 446pp. 